\title{
Sestamibi Scan
}

National Cancer Institute

\section{Source}

National Cancer Institute. Sestamibi Scan. NCI Thesaurus. Code C38091.

A nuclear imaging procedure which is performed to identify hyperparathyroidism.

Sestamibi is a nuclear radiopharmaceutical which is bound to the radioactive isotope

Tc99m. Tc99m-Sestamibi is taken up by both the thyroid gland and the parathyroid gland, and is also used in Nuclear Myocardial Perfusion Imaging. 